\title{
Fast-Handover Mechanism between 802.11 WLAN and 802.16 WiMax with MIH in PMIPv6
}

\author{
Cheol-Joong Kim • Seok-Cheon Park • Myung-Kyu Yi
}

Published online: 9 October 2013

(c) The Author(s) 2013. This article is published with open access at Springerlink.com

\begin{abstract}
As the wireless Internet services become widely available, users become able to use various Internet services without restriction in location. In particular, the demands on wireless Internet services are becoming greater, because mobile devices that support high mobility are getting smarter. However, if a user uses various wireless networks, much limitation occurs in network setting when they move a network different each other. This is because there are few appropriate handover mechanisms to support a heterogeneous network. We propose a fast-handover for heterogeneous networks that utilizes MIH in PMIPv6 to support heterogeneous networks and to reduce the handover latency time. And the performance evaluation for the proposed method was done separately for low speed and high speed mobility. The result presented shows that the suggested method has reduced latency time by $26 \%$ and packet losses by $90 \%$ (Avg.).
\end{abstract}

Keywords Handover · MIH · PMIPv6 · Fluid-flow

\section{Introduction}

The rapid development of Internet made it an essential component of every aspect of human existence beyond the simple practical use in personal life. In particular, the limitation of using Internet is getting less than before, because

\author{
C.-J. Kim · S.-C. Park $(\varangle) \cdot$ M.-K. Yi \\ Department of Computer Science, Gachon University, \\ SeongNam-Si, Kyungki-Do, 461-701, Republic of Korea \\ e-mail: scpark@gachon.ac.kr \\ C.-J. Kim \\ e-mail: huntcjk@empal.com \\ M.-K. Yi \\ e-mail: kainos@gachon.ac.kr
}

of the sustained development in wireless Internet technologies, such as, IEEE 802.11 WLAN and IEEE 802.16 WiMax and others. However, such technologies still have some limitations that cannot provide with seamless network service when the node moves in a heterogeneous network. And that come to demand other technologies that could let it move freely in a heterogeneous network and use the wireless Internet services continuously.

As a result, IEEE 802.21 MIH (Media Independent Handover) was proposed to offer the Internet services that could support handover in heterogeneous networks [1]. Therefore, users can be supported when they make the movement in various networks, such as, Cellular, WLAN, and WiMax. And users can access wireless Internet services without being aware of what kind of network is in use and where they are. Using $\mathrm{MIH}$, users who are on a $3 \mathrm{G}$ network can move onto WLAN that is cheaper than $3 \mathrm{G}$ networks when they get in WLAN network area without being aware of the moment the handover actually occurs. However, when the nodes perform handover procedure between networks with different technologies, there would be data packet losses and out of sequence data packets as it occurs in Mobile IPv4, IPv6 $[2,3]$. This is attributed to the difference between protocols in use and various access methods.

Also when many networks are linked, processing the signals generated by the handover becomes more complex. To overcome the problems, Fast-handover MIPv6 and Hierarchical MIPv6 were proposed but they still have limitation in mobility management because they are Host-based mobility protocol $[4,5]$.

So, we propose the Fast-Handover Mechanism for Heterogeneous Networks which is based on MIH in a networkbased mobility protocol Proxy MIPv6. Our objective is to reduce the handover latency time and data loss during handover. 
In this paper, we propose a Fast-Handover for Heterogeneous Networks that utilizes MIH in PMIPv6 to support heterogeneous networks, which can be used to evaluate its performance in terms of latency time and packet loss. The remainder of this article is organized as follows. In Sect. 2, we present related works. Section 3 we propose a FastHandover for Heterogeneous Networks that utilizes MIH in PMIPv6 to support heterogeneous network. Section 4 we propose an analytic model based on the fluid-flow mobility model, formulates the latency time and the packet loss using the analytic model, and presents various numerical results which show the latency time and packet loss. Section 5 concludes this paper.

\section{Related works}

\subsection{IEEE 802.21 MIH}

The standard provides information to allow handing over to and from cellular, GSM (Global System for Mobile communication), GPRS (General Packet Radio Service), WiFi, Bluetooth, IEEE 802.11 and IEEE 802.16 networks through different handover mechanisms. And IEEE 802.21 helps with Handover Initiation and Preparation, but handover execution is outside scope of IEEE 802.21. Moreover MIH does not define any new mobility management protocols. Figure 1 shows the structure and functions of MIH.

MIHF (MIH Function) that is the function of MIH is defined along the following classifications.

- MIES (Media Independent Event Service): Events originate within the link layer and destine to MIH Function within the local stack, or remote stack at the other end of the link, or both.

- MICS (Media Independent Command Service): To carry the upper layer decisions to the lower layers, and thus MICS controls the behavior of lower layers.

- MIIS (Media Independent Information Service): MIIS can help with network discovery and selection leading to more effective handover decision.

And several event messages that are mainly in each MIHF are listed as Table 1 [6].

\subsection{Proxy MIPv6}

Standardization of PMIPv6 is under progress in IETF NetLMM (Network-based Localized Mobility Management) WG. The PMIPv6 architecture consists of LMA (Localized Mobility Anchors) and MAG (Mobile Access Gateways). LMAs within the backbone network maintain a collection of routes for individual mobile nodes within the localized mobility management domain.

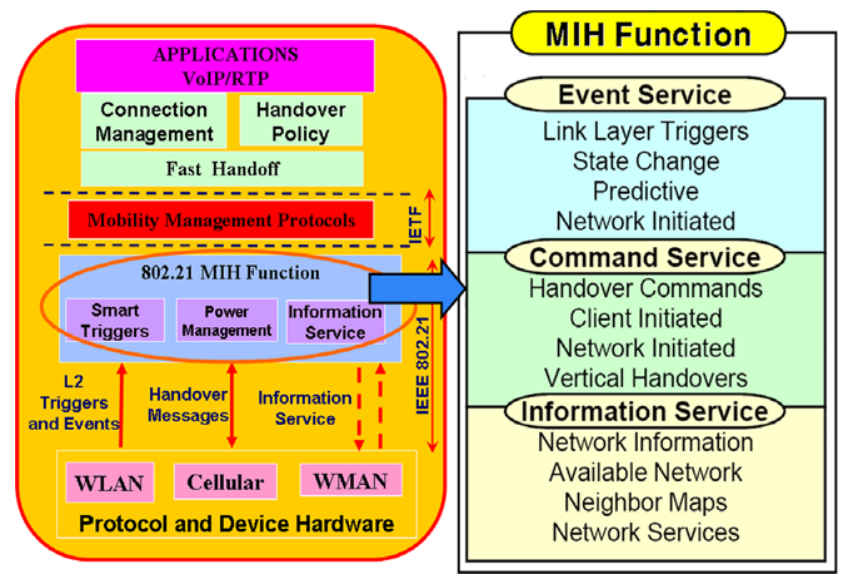

Fig. 1 Structure of MIH and MIH Function

Table 1 Event messages of MIH

\begin{tabular}{|c|c|c|}
\hline Event type & $\begin{array}{l}\text { Message name of } \\
\text { events }\end{array}$ & Description \\
\hline Predictive & Link_Going_Down & $\begin{array}{l}\text { Link Going Down L2 connection } \\
\text { breakdown imminent. }\end{array}$ \\
\hline $\begin{array}{l}\text { State } \\
\text { Change }\end{array}$ & Link_Detected & New L2 link has been found. \\
\hline $\begin{array}{l}\text { Handover } \\
\text { Command }\end{array}$ & $\begin{array}{l}\text { MIH_Handover_ } \\
\text { Initiate }\end{array}$ & $\begin{array}{l}\text { Initiates handovers and sends a list } \\
\text { of suggested networks and } \\
\text { suggested PoA (AP/BS). }\end{array}$ \\
\hline $\begin{array}{l}\text { Handover } \\
\text { Command }\end{array}$ & $\begin{array}{l}\text { MIH_Handover_- } \\
\text { Prepare }\end{array}$ & $\begin{array}{l}\text { This allows the client to query for } \\
\text { resources on new network and also } \\
\text { allows preparing the new network } \\
\text { for handover. }\end{array}$ \\
\hline
\end{tabular}

The routes point to the MAGs managing the links on which the mobile nodes currently are located. Packets for a mobile node are routed to and from the mobile node through tunnels between the LMA and MAG. When a mobile node moves from one link to another, the MAG sends a route update to the LMA [7].

PMIPv6 is possible to support mobility for IPv6 nodes without host involvement by extending Mobile IPv6 signaling messages between a network node and a home agent. This approach to supporting mobility does not require the mobile node to be involved in the exchange of signaling messages between itself and the home agent.

Because of the use and extension of Mobile IPv6 signaling and home agent functionality, a proxy mobility agent in the network performs the signaling with the home agent and does the mobility management on behalf of the mobile node attached to the network [8]. Figure 2 shows the basic components of PMIPv6.

Figure 3 shows the handover procedure of PMIPv6 that is similar to the access procedure of a mobile node on a PMIPv6 network. 


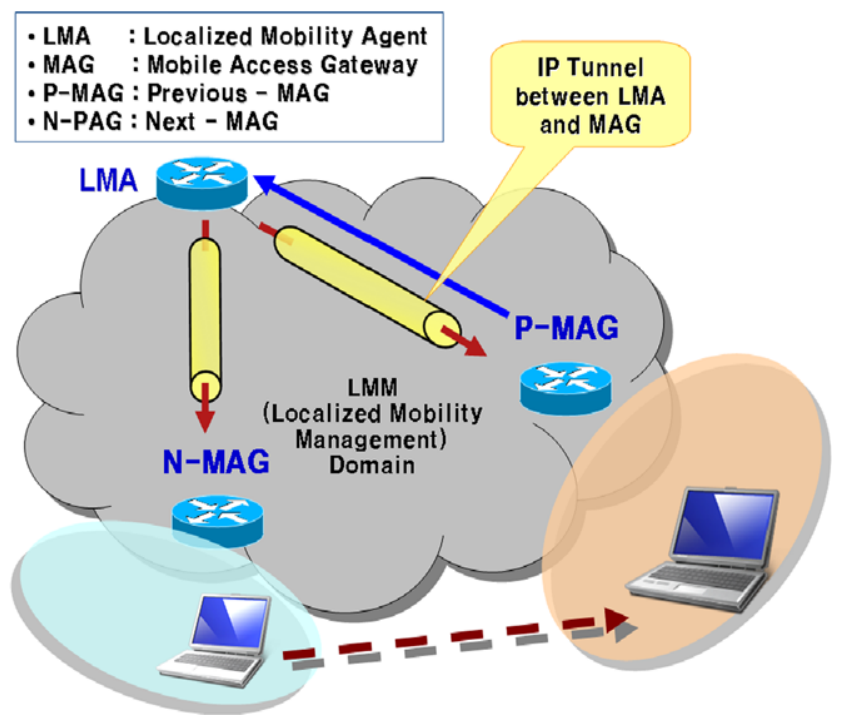

Fig. 2 Diagram of basic component of PMIPv6

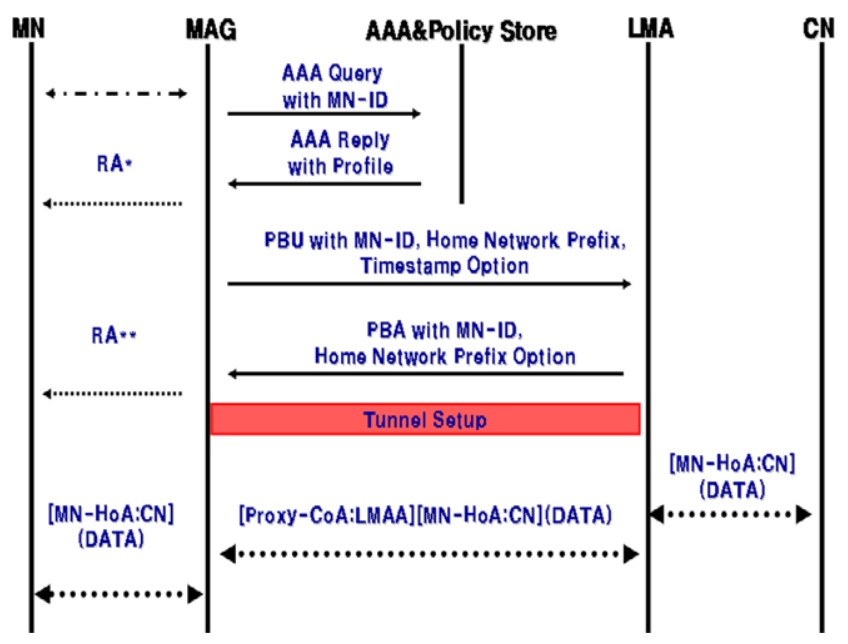

Fig. 3 Procedure of PMIPv6 handover

\section{Design of proposed Fast-Handover Mechanism}

\subsection{Scenario of proposal}

The objectives of the method proposed in this paper are the reception of data packets without disconnection and the reduction of handover delay during a handover of mobile node moving between different networks using PMIPv6. Other objectives are to minimize data packet loss and out-of-order receptions. Figures 4 and 5 describe the scenario for the proposed method.

The scenario assumes an IEEE 802.16 WiMax network and an IEEE 802.11 WLAN network based on the components of PMIPv6.

When moving from a P-MAG of an IEEE 802.16 WiMax network to a N-MAG of an IEEE 802.11 WLAN network, the information on the current AP (Access Point) and its

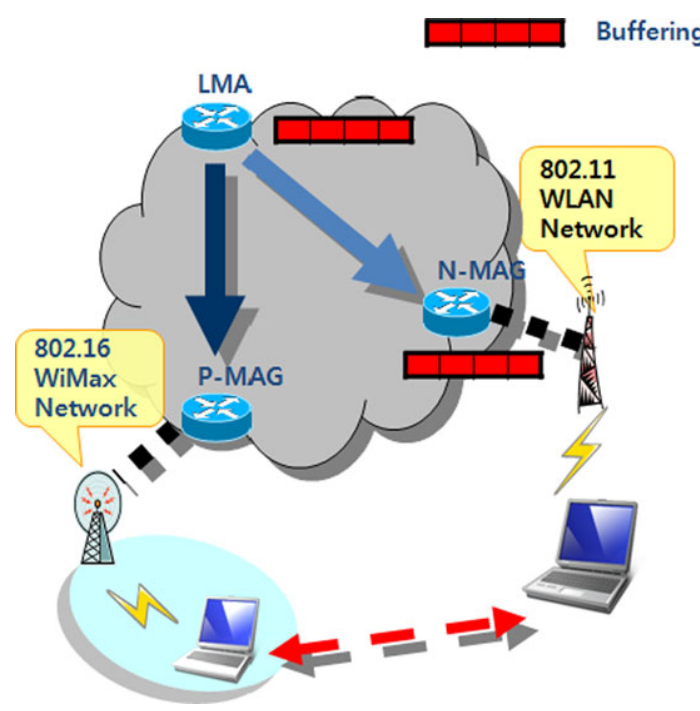

Fig. 4 Moving from 802.16 to 802.11

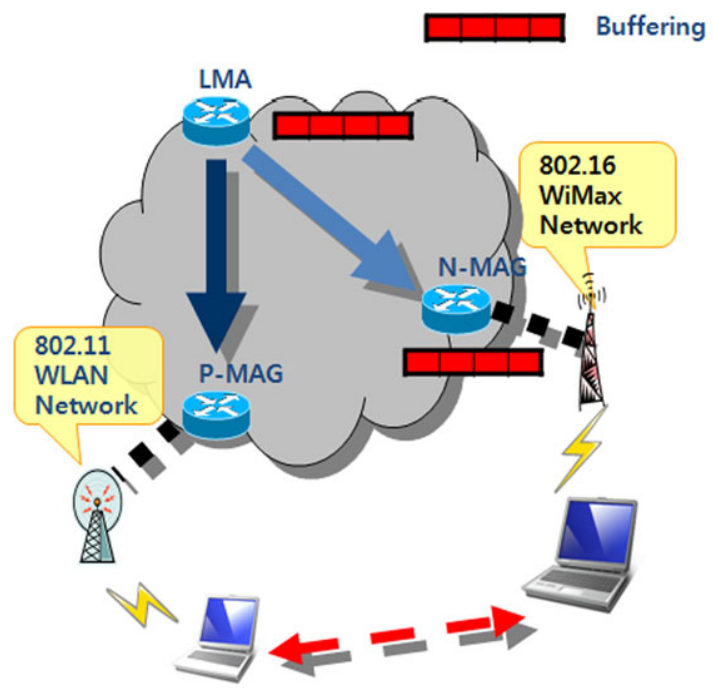

Fig. 5 Moving from 802.11 to 802.16

neighboring APs are collected through the Beacon Messages received from surrounding APs and utilized as the information on the AP to which the mobile node is handed over.

\subsection{Procedure for the proposed method}

\subsubsection{When moving from 802.16 to 802.11}

Figure 6 shows the procedure for the proposed method of handover when the mobile node is moving from an IEEE 802.16 WiMax network to an IEEE 802.11 WLAN network as described in Sect. 3.1.

When a mobile node attached to an IEEE 802.16 network detects the signal strength from the P-AP falling below the threshold value, it generates LINK_GOING_DOWN event of $\mathrm{MIH}$ and begins preparation for handover. 


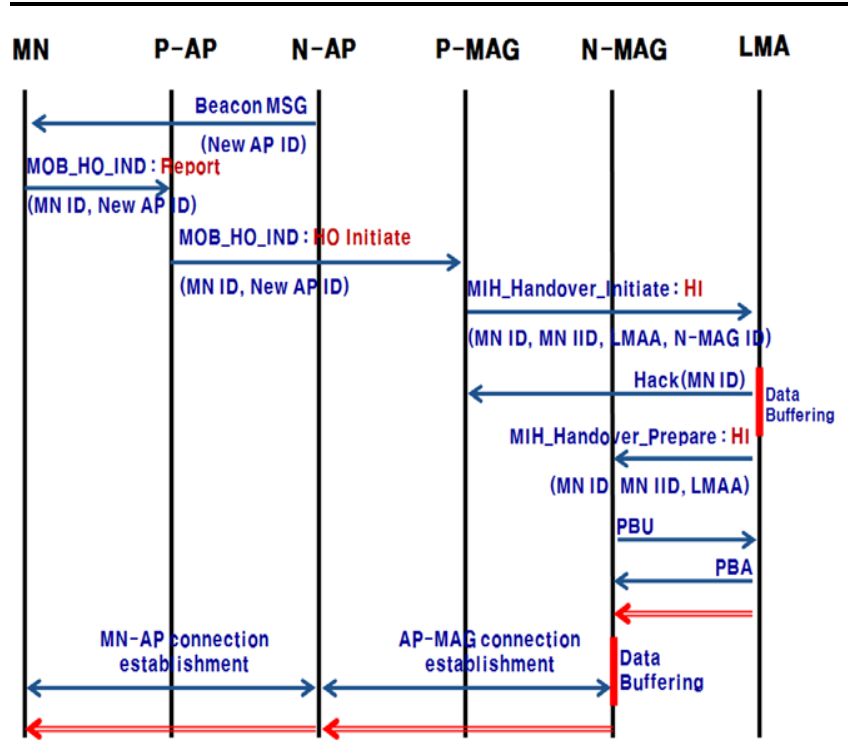

Fig. 6 Moving from 802.16 to 802.11

At the same time, the mobile node collects information on nearby IEEE 802.11 APs by receiving Beacon MSG from them. AP with best signal strength has higher priority because it is the most likely target to move to.

Then the mobile node sends MAC (Media Access Control) information of N-AP to P-AP using Target_BS_ID slot of MOB_HO_IND message defined in IEEE 802.16.

On receiving the message from the mobile node, P-AP sends MOB_HO_IND (MAC: N-AP) message to P-MAG which in turn sends MAC information of the mobile node in a MIH_Handover_Initiate message, a MIH command message, to LMA.

From then on, LMA buffers data to be sent to P-MAG until PMIPv6 binding update is completed with N-MAG. At the same time, LMA sends MAC and other relevant information of the mobile node to be handed over to N-MAG on a MIH_Handover_Prepare message and execute Proxy Binding Update with N-MAG.

After binding update is finished, LMA sends buffered data to N-MAG which in turn buffers the data until the node is connected. When the node is registered with N-MAG, it receives buffered data from N-MAG.

\subsubsection{When moving from 802.11 to 802.16}

Figure 7 illustrates the procedure of a handover of a mobile node moving from an IEEE 802.11 WLAN network to an IEEE 802.16 WiMax network.

When a mobile node attached to an IEEE 802.11 network detects the signal strength from the P-AP falling below the threshold value, it generates LINK_GOING_DOWN event of MIH and begins preparation for handover.

At the same time, the mobile node collects information on nearby IEEE 802.16 APs by receiving MOB_NBR_ADV

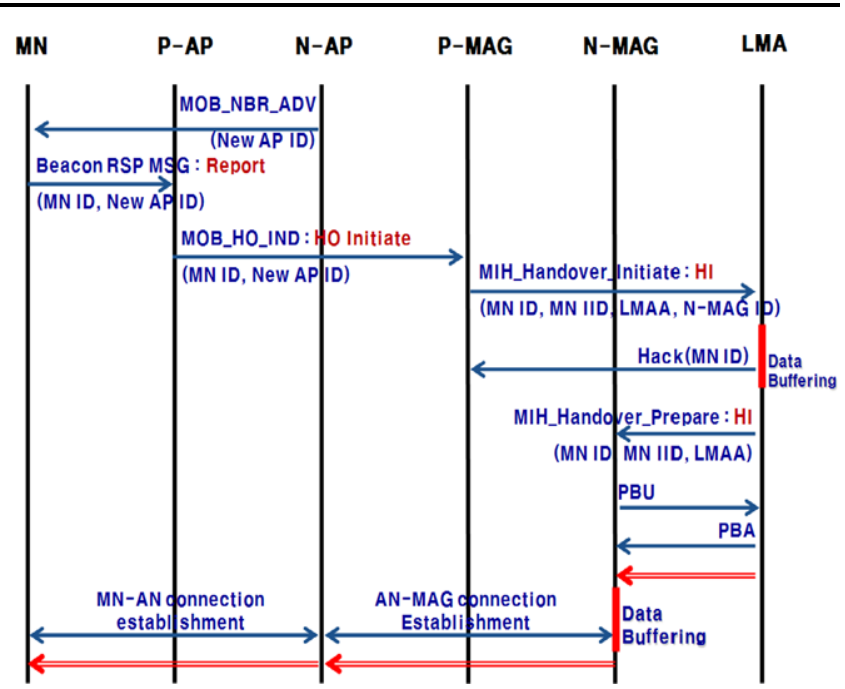

Fig. 7 Moving from 802.11 to 802.16

from them. AP with best signal strength has higher priority because it is the most likely target to move to.

Then the mobile node sends the MAC information of NAP to P-AP on a Beacon RSP message. P-AP in turn sends the information to P-MAG. P-MAG sends MIH_Handover_ Initiate message with the MAC information of the mobile node to LMA.

From then on, LMA buffers data to be sent to P-MAG until PMIPv6 binding update is completed with N-MAG. Rest of the procedure is the same as that of moving from IEEE 802.16 to IEEE 802.11

\section{Analysis of proposed mechanism}

To analyze performance of proposed mechanism, fluid flow model was applied in this paper. So, we compare existing handover procedure of PMIPv6 with our suggestion on handover latency time and loss of data packets.

\subsection{Modeling structure}

We suppose a hexagonal model as Fig. 8 and the $N(R)$; number of all cells, comes from Eq. (1) [9].

The cell boundary crossing rate $R_{c}$ (Eq. (2)) where $R_{c}$ is the cell crossing rate (mobiles/s); $\rho$ is the mobile density (mobiles $\left./ \mathrm{m}^{2}\right) ; v$ is the moving velocity $(\mathrm{m} / \mathrm{s}$ ); and $L$ is the cell perimeter $(\mathrm{m})$ [9].

$$
\begin{aligned}
& N(R)=\sum_{r=1}^{R} 6 r+1=3 R(R+1)+1 \\
& R_{c}=\frac{\rho v L_{c}}{\pi}
\end{aligned}
$$

Mobile devices move across a boundary in two directions. For evaluation purposes, however, only one direction 


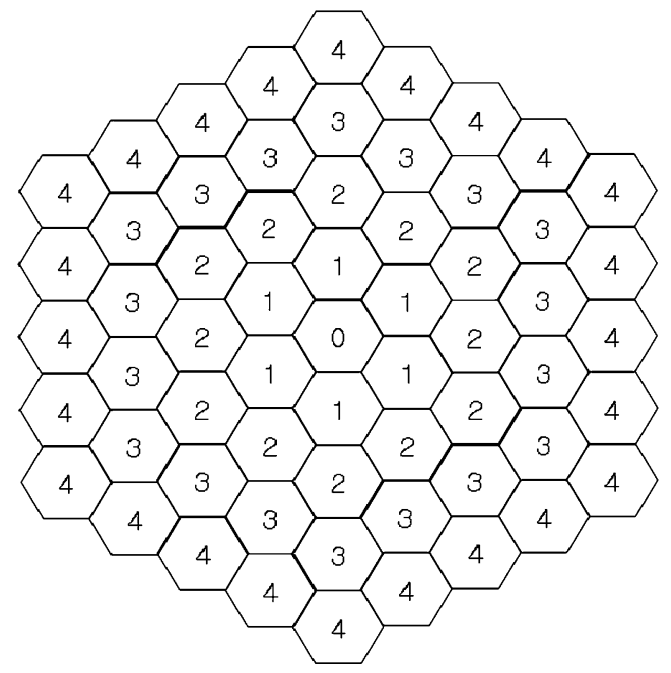

Fig. 8 Structure of suggestion mobility model

needs to be considered. The paging area boundary crossing rate $R_{d}$ is

$R_{d}=\frac{\rho v L(R)}{\pi}$

And Formula (4) means equation for $L(R)$.

$L(R)=6 \times(2 R+1) \times \frac{L_{c}}{6} \quad(R \geq 1)$

\subsection{Evaluation of proposed mechanism}

In this paper, we compare existing handover method under PMIPv6 and the proposed handover method. Overall handover delay is defined as the time from L2 handover initiation to the completion of location registration by the mobile node.

All the participants of a handover, i.e. mobile node, MAG, LMA, HA, and CN are loaded with MIH stack. The relatively small control message transmission delay as well as the processing delay at each node is not considered. Parameters for the performance evaluation of the proposed method are defined as follows.

- Moving from IEEE 802.16 to IEEE 802.11

- $T_{N A P 1}$ : Transmission delay to send MOB_HO_IND (N-AP, MN) message from mobile node to P-AP

- $T_{N A P 2}$ : Transmission delay to send MOB_HO_IND (N-AP, MN) message from P-AP to P-MAG

- Moving from IEEE 802.11 to IEEE 802.16

- $T_{N A P 1}$ : Transmission delay to send RSP(N-AP, MN) message from mobile node to P-AP

- $T_{N A P 2}$ : Transmission delay to send RSP(N-AP, MN) message from P-AP to P-MAG

- Common parameters

- $T_{L 2}$ : L2 handover delay at the mobile node
- $T_{A P 1}$ : Transmission delay to send Attach message from mobile node to AP in PMIPv6

- $T_{A P 2}$ : Transmission delay to send Attach message from AP to MAG

- $T_{R A 1}$ : Transmission delay to send RA message from MAG to AP

- $T_{R A 2}$ : Transmission delay to send RA message from AP to mobile node

- $T_{A R E}$ : Transmission delay to send authentication request message from MAG to AAA (Authentication, Authorization and Accounting) server

- $T_{A R P}$ : Transmission delay to send authentication reply message from AAA server to MAG

- $T_{H I}$ : Transmission delay to send MIH_Handover Initiate (MN MAC info) message from P-MAG to LMA

- $T_{H P}$ : Transmission delay to send MIH_Handover_ Prepare (N-AP, MN) message from LMA to N-MAG

- $T_{P B U}$ : Proxy Binding Update message transmission delay

- $T_{P B A}$ : Proxy Binding Ack. message transmission delay

The handover delay $T_{H O}$ in PMIPv6 and in the proposed method can be expressed as Eqs. (5) and (6), respectively.

- PMIPv6:

$$
\begin{aligned}
T_{H O}= & T_{L 2}+T_{A P 1}+T_{A P 2}+T_{A R E}+T_{A R P}+T_{R A 1} \\
& +T_{P B U}+T_{P B A}+T_{R A 2}
\end{aligned}
$$

- Proposed method:

$T_{H O}=T_{L 2}+T_{N A P 1}+T_{N A P 2}+T_{H I}+T_{H P}+T_{P B U}+T_{P B A}$

If we apply these to the Fluid Flow model, the total handover delay $T$ and the packet loss $L$ can be derived as Eqs. (7) and (8). N(A) is the area of a Cell and $P$ is the packet size.

$T=\frac{p N(A)}{N(R) R_{C}-R_{d}} T_{H O}$

$L=T \cdot P$

\subsection{Performance analysis of the proposed method}

In this paper, we evaluated handover performance at low speed and high speed separately. It is because that both 802.11 WLAN which supports $5 \sim 30 \mathrm{~km} / \mathrm{hr}$ and 802.16 WiMax which supports over $100 \mathrm{~km} / \mathrm{hr}$ must be considered. The parameter values for the analysis were referenced from $[10,11]$ and [12]. They are shown in Table 2. When parameter values are applied to Eq. (7), the resulting handover delay 
Fig. 9 Latency time vs. velocity at low speed

Fig. 10 Latency time vs. velocity at high speed
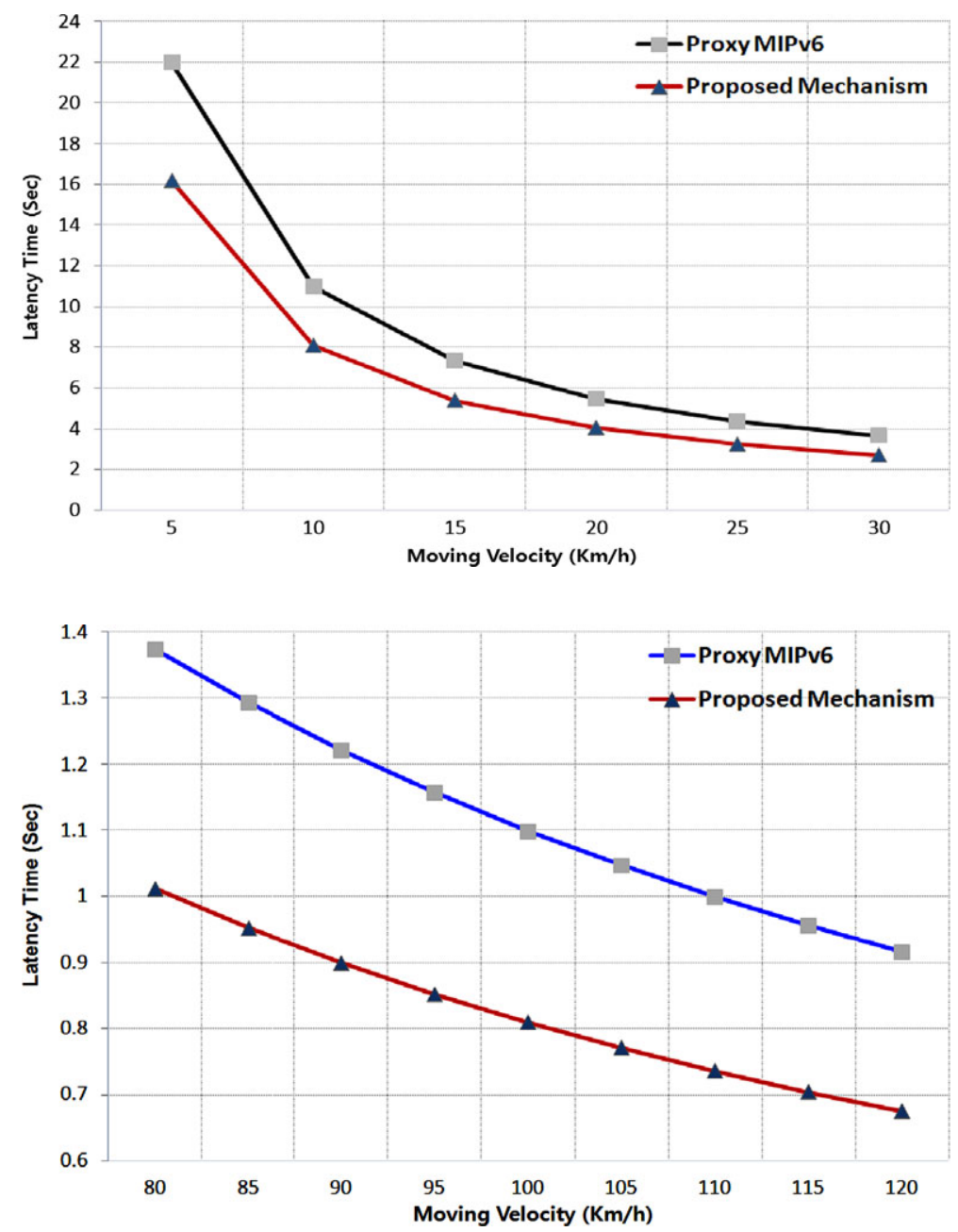

Table 2 System parameters for numerical analysis

\begin{tabular}{lllll}
\hline$\rho$ & $v$ & $L(C)$ & $N(A)$ & $P$ \\
\hline 0.02 & $5 \sim 30,80 \sim 120$ & 36 & 120 & 50 \\
\hline
\end{tabular}

can be seen in Figs. 9 and 10. First, the Fig. 9 is the plot of handover delays at low speed.

On the other hand, IEEE 802.16 supports mobility at over $100 \mathrm{~km} / \mathrm{hr}$. Figure 10 shows the handover delay when the mobile node moves at relatively high speed.

The method proposed in this paper achieved maximum $26 \%$ reduction in handover delay compared to the existing method, as can be seen in Figs. 9 and 10. Especially, our method simplified the handover procedure by loading information about the mobile node on MOB_HO_IND message and transmitting it to LMA and N-MAG beforehand, thus eliminating the need to exchange messages with AAA server to acquire mobile node profile and authentication.

Packet loss was reduced up to $90 \%$ by shortening the handover delay as shown in Figs. 11 and 12 when the packet size was assumed to be 50 bytes. While data packets are

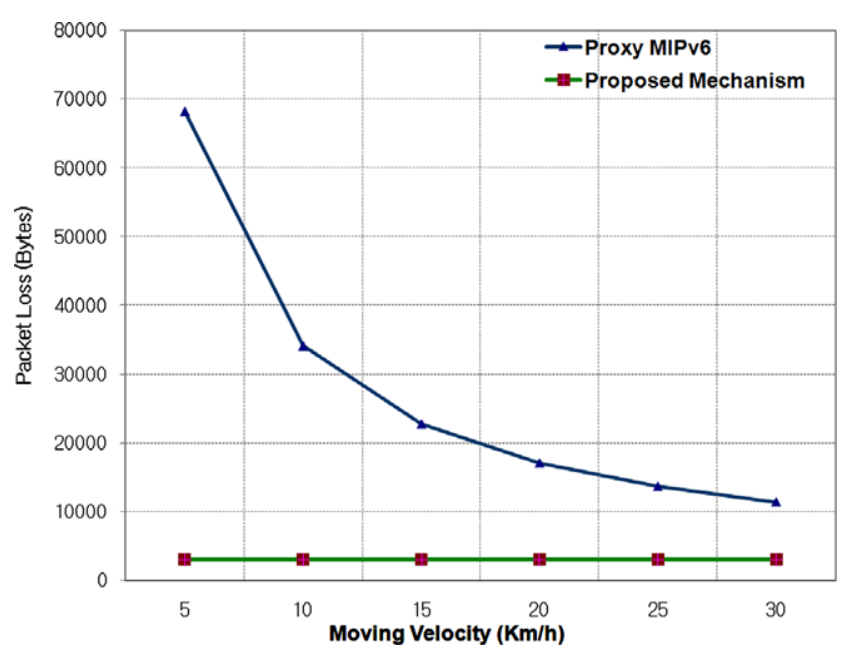

Fig. 11 Amount of packet loss at low velocity

lost during the handover between the mobile node and $\mathrm{N}$ MAG in PMIPv6, LMA and N-MAG buffers data during LMA's reception of MIH_Handover_Initiate message in the proposed method, thus reducing data loss during handover. 


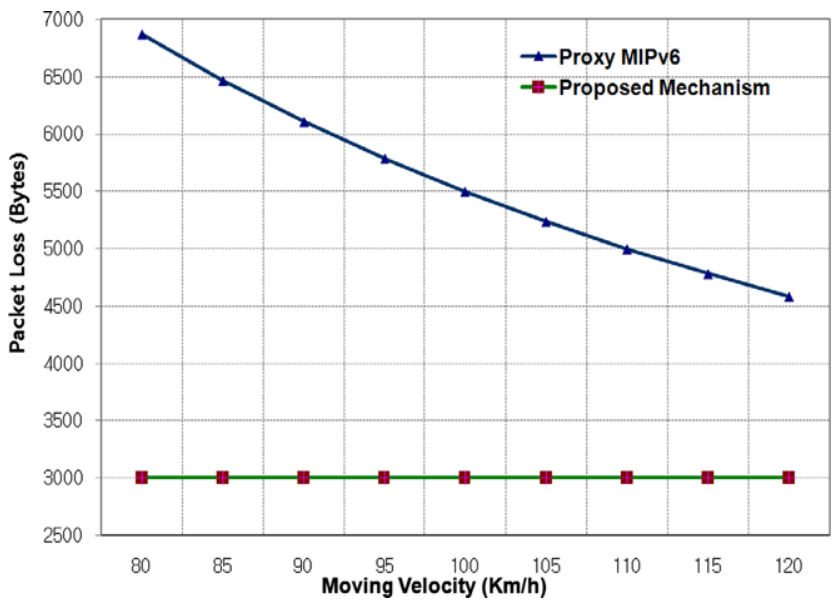

Fig. 12 Amount of packet loss at high velocity

\section{Conclusion}

In this paper, we proposed an improved method for handover in a heterogeneous network based on PMIPv6. Proposed method is more efficient than the existing method for handover between IEEE 802.16 WiMax network and IEEE 802.11 WLAN in PMIPv6 environment using IEEE 802.21 MIH.

Proposed method utilizes MAC information of nearby APs through Beacon message provided by IEEE 802.11 WLAN environment. It also supports fast handover by utilizing LINK_GOING_DOWN message in MIH. When the mobile node moves to a neighboring MAG, binding update between LMA and N-MAG is completed beforehand, and data are buffered in advance enabling fast packet transmission.

In this paper, the performance evaluation for the proposed method was done separately for low speed and high speed mobility. Using the proposed method, handover delay was reduced maximum $26 \%$ from existing PMIPv6 handover delay. Data packet loss was reduced by up to $90 \%$ as well.

Acknowledgements This research was supported by MSIP (the Ministry of Science, ICT and Future Planning), Korea, under the IT-CRSP (IT Convergence Research Support Program) (NIPA-2013H0401-13-1001) supervised by the NIPA (National IT Industry Promotion Agency).

Open Access This article is distributed under the terms of the Creative Commons Attribution License which permits any use, distribution, and reproduction in any medium, provided the original author(s) and the source are credited.

\section{References}

1. IEEE 802.21 (2008). Draft standard for local and metropolitan area networks: media independent handover services. December, 2008.
2. Johnson, D., Perkins, C., \& Arkko, J. (2011). Mobility support in IPv6. IETF RFC 3775, Mar 11, 2011.

3. Perkins, C. (Ed.) (2002). IP mobility support in IPv4. IETF RFC 3344, August, 2002.

4. Koodli, R. (Ed.) (2005). Fast handovers for mobile IPv6. IETF RFC 4068, July 2005.

5. Soliman, H., Castelluccia, C., El Malki, K., \& Bellier, L. (2005). Hierarchical mobile IPv6 mobility management. IETF RFC 4140, Aug 2005.

6. http://www.ieee802.org/21/, 802.21 tutorial

7. Kempf, J. (Ed.) (2007). Problem statement for network-based localized mobility management (NETLMM). IETF RFC 4830, April 2007.

8. Gundavelli, S. (Ed.) (2008). Proxy mobile IPv6. IETF RFC 5213, August, 2008.

9. Akyildiz, I. F., \& Wang, W. (2002). A dynamic location management scheme for next-generation multitier PCS systems. IEEE Transactions on Wireless Communications, 1(1), 178-189.

10. Zhang, X., Castellanos, J., \& Capbell, A. (2002). P-MIP: paging extensions for mobile IP. Mobile Networks and Applications, 7(2), $127-141$.

11. Stephane, A., \& Aghvami, A. H. (2001). Fast handover schemes for future wireless IP networks: a proposal and analysis. In IEEE vehicular technology conference, May 2001, (Vol. 3, pp. 20462050).

12. Sangheon, P., \& Yanghee, C. (2004). A Study on Performance of Hierarchical Mobile IPv6 in IP-Based Cellular Networks. IEICE Transactions on Communications E, 87-B(3).

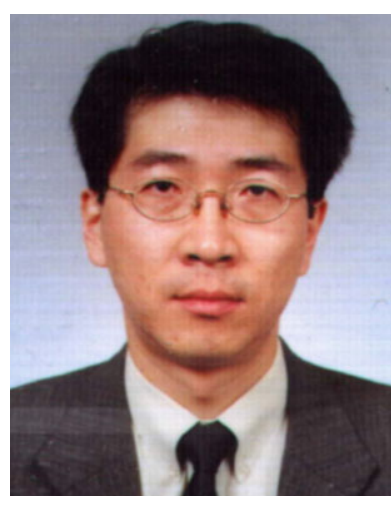

Cheol-Joong Kim received B.S. (1992) and M.S. (1995) degree from Kyungwon University, both in Computer Science. He is currently working toward a Ph.D. degree in Computer Science at the Kyungwon University. From 1995 to 2002, he was with Korea Telecom International, Solvix Technology, and Daou Tech., where he contributed to the research and development of Electronic Data Interchange system, WAP, and Wireless Internet system. His research interests include mobile computing, ubiquitous sensor networks, and next-generation wireless networks.

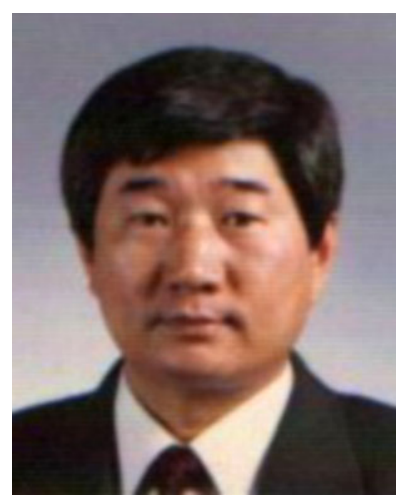

Seok-Cheon Park received his B.S. degree in Electronic Engineering from Korea University in 1977, and his M.S. degree in Computer Engineering from Korea University in 1982, and Ph.D. degree in Computer Engineering from Korea University in 1989. He was with LG R\&D Center during 19791985, where he served as director of Data Communication Section. He worked as a Post Doctoral researcher at the University of California, Irvine, during 1991-1992. Since 1988, he has been with the College of IT, Kyungwon University, where he is currently a Professor. His research interests include wireless networks, high speed communication protocol, and ubiquitous computing. 


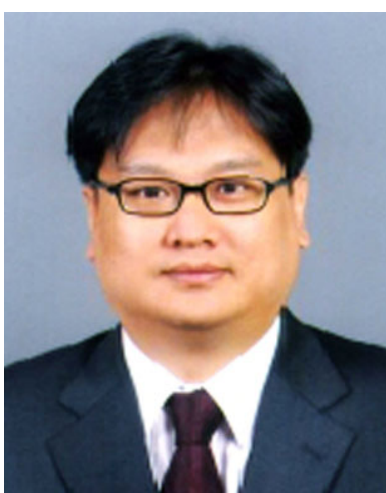

Myung-Kyu Yi received his B.S. degree in Computer Science from Suwon University in 1997, and his M.S. degree in Computer Science from Soongsil University in 1999. $\mathrm{He}$ received the Ph.D. degree in Computer Science and Engineering from Korea University in 2005. Since 2006, he has been a BK21 Research Professor with the College of IT at Kyungwon University, Korea. His research interests include mobility management, wireless sensor networks, and network security. 